\title{
Plastia inguinal asistida por robot: reporte de 98 casos realizados en México
}

\author{
Robot-assisted inguinal hernia repair: report of 98 cases performed in Mexico \\ Javier Alberto Kuri Osorio, ${ }^{*}$ Karla Ivonne Luján Mendoza, ${ }^{*}$ Francisco Iván Galeana Nogueda, ${ }^{*}$ \\ Juan José Solorzano Adame, ${ }^{*}$ Manuel Preben Aguirre García* \\ * Centro de Cirugía Robótica. Hospital Ángeles Pedregal. Ciudad de México.
}

\section{RESUMEN}

Objetivo: Describir la experiencia en torno a los abordajes transabdominal preperitoneal (TAPP) y Stoppa modificado, ambos asistidos por robot de un mismo equipo quirúrgico, así como demostrar las ventajas del abordaje robótico. Material y métodos: Se realizó un estudio prospectivo, descriptivo, observacional y longitudinal de pacientes intervenidos quirúrgicamente con las técnicas r-TAPP y r-Stoppa modificados para hernias inguinales unilaterales y bilaterales respectivamente, durante el periodo comprendido entre enero de 2016 y agosto de 2020, en los hospitales Ángeles Pedregal y ABC Santa Fe en la Ciudad de México. Se analizaron las características clínicas, la técnica quirúrgica y su resultado. Resultados: Fueron incluidos 98 pacientes, 35 mujeres (35.71\%) y 63 hombres (64.28\%). La mediana de edad fue de 52.3 años. El tiempo aproximado de docking fue de 4 minutos. Setenta y tres pacientes presentaron hernia inguinal unilateral $(74.48 \%)$, se utilizó la técnica r-TAPP, el tiempo quirúrgico medio fue de 46.3 minutos. Veinticinco pacientes presentaron hernia inguinal bilateral $(25.51 \%)$, se recurrió a la técnica rStoppa modificada, el tiempo quirúrgico medio fue de 72.3 minutos. En el seguimiento posoperatorio, la única complicación que se ha presentado es seroma en herida umbilical. Hasta el momento, ningún paciente ha presentado recidiva. Conclusión: La cirugía asistida por robot ha ido cambiando progresivamente dichos procedimientos quirúrgicos. Las técnicas r-TAPP y r-Stoppa modificada son reproducibles, seguras y efectivas a corto plazo para pacientes con hernias inguinales unilaterales y bilaterales. Esta serie, realizada por un solo equipo quirúrgico, es la más grande reportada hasta el momento en México.

Palabras clave: Cirugía robótica, hernia inguinal, sistema robótico Da Vinci, hernia inguinal bilateral.

\section{ABSTRACT}

Objective: To describe the experience with the preperitoneal transabdominal approach (TAPP) and modified Stoppa approach, both executed by the same surgical team and assisted by robot, as well as to demonstrate the advantages of the robotic approach. Material and methods: A prospective, descriptive, observational and longitudinal study of patients who underwent surgery with the r-TAPP and modified $r$-Stoppa techniques, for unilateral and bilateral inguinal hernias, respectively, was conducted during the period between January 2016 and August 2020, at the Hospital Ángeles Pedregal and ABC Santa Fe hospital in Mexico City. The clinical characteristics, the surgical technique and its results were analyzed. Results: 98 patients were included, 35 women (35.71\%) and 63 men (64.28\%). The median age was 52.3 years. The approximate docking time was 4 minutes. Seventy-three patients presented unilateral inguinal hernia (74.48\%), the r-TAPP technique was performed, the mean surgical time was 46.3 minutes. Twenty-five patients presented bilateral inguinal hernia (25.51\%), a modified r-Stoppa technique was performed, the mean surgical time was 72.3 minutes. In the postoperative follow-up, the only complication that has occurred is an umbilical wound seroma. So far, no patient has relapsed. Conclusion: Robotic-assisted surgery has progressively changed surgical procedures. The r-TAPP and modified $r$-Stoppa techniques are, in the short term, reproducible, safe and effective techniques for patients with unilateral and bilateral inguinal hernias. This series is the largest reported so far in México, carried out by a single surgical team.

Keywords: Robotic surgery, inguinal hernia, DaVinci robotic system, bilateral inguinal hernia. 


\section{INTRODUCCIÓN}

La primera reparación de hernia inguinal con mínimo acceso se realizó en el año de 1979 cuando P. Fletcher introdujo la técnica laparoscópica. En 1990 se realizó la primera plastia inguinal laparoscópica por Ger y colaboradores, usando el enfoque clásico de la reparación preperitoneal. Las plastias inguinales representan cerca de 500,000 cirugías por año en Estados Unidos, y es uno de los procedimientos más comunes entre los cirujanos generales. ${ }^{1,2}$

Las técnicas de mínimo acceso ofrecen reducción del dolor, disminución de las infecciones en el sitio quirúrgico, estancia hospitalaria más corta y recuperación funcional rápida. El incremento en la incorporación de la cirugía robótica, en todas las especialidades quirúrgicas, ofrece otro enfoque para esos procedimientos que se realizan con frecuencia. ${ }^{3}$

La técnica robótica fue descrita por primera vez en la literatura por Escobar Domínguez y colegas en 2015. La reparación robótica de la hernia inguinal es la progresión natural de este proceso, utilizando esos mismos principios operativos, pero con avances críticos que cambian tanto la experiencia del paciente como del cirujano. ${ }^{4-6}$ Para el cirujano, la cirugía robótica permite una vista tridimensional del campo de operación al tiempo que proporciona una muñeca articulada, lo que mejora enormemente la experiencia ergonómica de las tradicionales «pinzas rectas» utilizadas en cirugía laparoscópica. ${ }^{7-9}$

El objetivo de este trabajo es describir la experiencia de un mismo equipo quirúrgico ejecutando los abordajes transabdominal preperitoneal (TAPP) y Stoppa modificado, ambos asistidos por robot. Este estudio es el primero en México, y muestra resultados tempranos asociados con el sistema quirúrgico Da Vinci $\mathrm{Si}^{\circledR}$, empleando la técnica transabdominal preperitoneal asistida por robot (r-TAPP) en el caso de hernias inguinales unilaterales y r-Stoppa modificado en hernias inguinales bilaterales. Es importante enfatizar las ventajas del abordaje robótico, como visualización tridimensional, pinzas articuladas, visión con zoom 10×, disminución del riesgo de lesión nerviosa y vascular, aumento de la destreza y neutralización del temblor fino permitiendo realizar cirugías mucho más complejas con mayor precisión.

\section{MATERIAL Y MÉTODOS}

Serie de casos consecutivos que comprende una cohorte, y estudio descriptivo, observacional y longitudinal de pacientes intervenidos quirúrgicamente empleando las técnicas r-TAPP y r-Stoppa modificada para hernias inguinales unilaterales y bilaterales respectivamente, durante el periodo comprendido entre enero de 2016 y agosto de 2020, en los hospitales Ángeles Pedregal y ABC Santa Fe en la Ciudad de México, por un mismo grupo quirúrgico y liderado por un solo cirujano. Se consideraron pacientes con diagnóstico de hernia inguinal unilateral o bilateral, evidenciadas clínicamente y confirmadas mediante la realización de ultrasonidos dinámicos de pared abdominal.

Todos estos pacientes fueron ingresados cumpliendo un protocolo preoperatorio completo y permisible, donde se evaluaron comorbilidades y se obtuvo la firma de consentimiento informado.

Los criterios de inclusión fueron los siguientes: diagnóstico de hernia inguinal unilateral o bilateral con o sin cirugías previas, pacientes con o sin obesidad. Se excluyeron a aquellos pacientes que presentaran estrangulación al momento del diagnóstico.

En todos los pacientes se utilizó profilaxis antibiótica con cefalotina 1 gramo intravenoso, una hora previa al procedimiento quirúrgico. En las primeras 20 cirugías se realizó colocación de sonda transuretral, previo a la realización del procedimiento quirúrgico; tras el dominio de la técnica quirúrgica a partir del caso 21, sólo se les solicitó a tales pacientes que previo al procedimiento presentaran una micción, manteniendo vaciamiento vesical preoperatorio. Todos los pacientes fueron intervenidos con anestesia total endovenosa (TIVA).

Los pasos fundamentales del procedimiento quirúrgico son: colocación del paciente en decúbito dorsal, posterior a anestesia total endovenosa (TIVA). Para ingresar a la cavidad abdominal, se realiza una incisión transumbilical de aproximadamente $1.2 \mathrm{~cm}$, se incide la aponeurosis bajo visión directa y se coloca trocar transumbilical de $12 \mathrm{~mm}$ (la cámara siempre se coloca en la cicatriz umbilical), se realiza la insuflación de la cavidad abdominal con $\mathrm{CO}_{2}$ a $12 \mathrm{mmHg}$, donde ulteriormente se introduce la cámara a $30^{\circ}$, el siguiente paso es colocar dos trocares más, de 8 $\mathrm{mm}$ cada uno, a $10 \mathrm{~cm}$ del trocar transumbilical. Después, se coloca el paciente en posición de Trendelemburg y se realiza side docking robótico, el cual dura 4 minutos aproximadamente, siempre se realiza conservando las piernas cerradas y de esta forma no se modifica la anatomía de la región inguinal. Posteriormente, se introducen bajo visión directa pinza Prograsp y gancho monopolar en cada uno de los brazos robóticos. En ambas técnicas quirúrgicas (r-TAPP y r-Stoppa modificado) la colocación de dichos trocares es la misma y en línea recta.

La técnica r-TAPP se inicia realizando una incisión en el peritoneo, en el plano avascular que se forma entre el peritoneo y la fascia transversalis. La fascia transversalis debe permanecer ventral, para proteger los vasos epigástricos. Medialmente, la disección se lleva a cabo en el espacio de Retzius para exponer el ligamento de Cooper, luego, se realiza la reducción del saco herniario. Para realizar la disección del saco herniario y el cordón espermático, se 
utiliza disección roma y gancho monopolar. En la técnica r-Stoppa, la modificación consiste en que la disección peritoneal se realiza 1-2 cm por debajo de la línea arcuata. En aquellos casos de hernias inguinoescrotales, donde el defecto herniario tiende a ser mayor de $1 \mathrm{~cm}$, éste se cierra utilizando sutura barbada absorbible y se plica la fascia transversalis, con esto se disminuye el riesgo de formación de seroma.

La malla utilizada en la plastia inguinal unilateral es Progrip (conformada por polipropileno monofilar y microfibras de ácido poliláctico que tienen efecto velcro); en todos los casos, tanto la disección como la colocación de la malla sobrepasan la línea media para disminuir el riesgo de recidiva.

En la plastia inguinal bilateral se utiliza malla UltraPro (conformada a partes iguales por fibras de polipropileno no absorbible y fibras de poliglecaprona absorbible). De acuerdo con las guías de International Endohernia Society, la recomendación en el tamaño de la malla es de $10 \times 15$ $\mathrm{cm}$, y se coloca una malla de cada lado, las cuales deben tener una superposición (overlap) en la línea media para disminuir el riesgo de migración, sin embargo, aquí se utilizó como medida estándar $15 \times 12 \mathrm{~cm}$ y en lugar de colocar dos mallas, se utilizó una sola malla lo suficientemente ancha de forma longitudinal, horizontal y vertical de $30 \times 12 \mathrm{~cm}$ para disminuir dicho riesgo.

En ambas técnicas quirúrgicas, la malla se fija con puntos simples de sutura absorbible al ligamento de Cooper y, en el caso del r-Stoppa, a pared abdominal $2 \mathrm{~cm}$ medial a los vasos epigástricos y en el borde lateral de la malla, evitando aquellos nervios de la región inguinal en el abordaje posterior, así como los vasos iliacos. Se realiza el cierre del colgajo peritoneal empleando sutura barbada 3-0 (V-Lo). El usar sutura absorbible, y la fijación de los puntos simples que no se afrontan al máximo, así como la separación entre cada punto de $3 \mathrm{~mm}$, reducen de manera significativa el tiempo quirúrgico y causa menor dolor posoperatorio que la colocación de tackers.

El posoperatorio se maneja administrando analgésicos intravenosos como ketoprofeno de 100 mg cada 8 horas, paracetamol 1 gramo cada 6 horas, y se continúa con cefalotina de 1 gramo cada 12 horas, además, se colocan compresas de hielo en la región inguinal durante las primeras 24 horas. Todos los pacientes fueron egresados del hospital a las 24 horas de haber realizado el procedimiento quirúrgico.

Se realizó la evaluación posoperatoria en consulta a los siete días, 30 días, seis meses y un año del procedimiento quirúrgico. El seguimiento consiste únicamente en evaluación clínica. El reinicio de la actividad física es gradual, el ejercicio se retoma cuatro semanas posteriores al procedimiento quirúrgico.

\section{RESULTADOS}

Durante el periodo de estudio fueron incluidos 98 pacientes, 35 mujeres (35.71\%) y 63 hombres (64.28\%). La media de edad fue de 52.3 años (el rango de edad fue entre 19-88 años). Sólo ocho pacientes presentaban comorbilidades como diabetes mellitus e hipertensión arterial sistémica (8.16\%). Diez pacientes con antecedente de tabaquismo (10.2\%). Siete de los pacientes presentaban obesidad (7.14\%). El tiempo de docking aproximado es de 4 minutos.

De aquellos 98 pacientes incluidos en este estudio, $73(74.48 \%)$ presentaron hernia inguinal unilateral y 25 bilateral (25.51\%), representando un total de 123 regiones inguinales tratadas mediante abordaje quirúrgico asistido por robot. Como se expone en la Tabla 1, se abordaron diferentes tipos de hernias (directas, indirectas, femorales y mixtas), dentro de las 71 hernias inguinales indirectas se incluyen ocho hernias inguinoescrotales en las que se encontraba dilatado el anillo inguinal profundo con un rango de $1.5-3 \mathrm{~cm}$.

Todos los pacientes que padecían hernias inguinales unilaterales fueron tratados mediante r-TAPP, el tiempo quirúrgico medio fue de 46.3 minutos (rango de 35-120 minutos) y aquellos pacientes con hernia inguinal bilateral (25.51\%) mediante r-Stoppa modificada, el tiempo quirúrgico medio fue de 72.3 minutos (rango de 50-135 minutos). El sangrado en ambas técnicas quirúrgicas se cuantificó en menos de $10 \mathrm{~mL}$.

De los cuatro casos de hernias inguinales recidivantes, uno fue por recurrencia bilateral previamente tratado con plastia bilateral laparoscópica usando colocación de malla y recurrencia bilateral, dos pacientes tenían antecedente de plastia inguinal bilateral laparoscópica con recurrencia unilateral y un paciente con antecedente de plastia con abordaje anterior abierto, sin colocación de malla y recurrencia ipsilateral. Al igual que para esos casos de hernias primarias, para las recidivantes, en el caso de una recidiva bilateral ésta se resolvió mediante un procedimiento de rStoppa con colocación sin complicaciones, mostrando un tiempo quirúrgico de 85 minutos y ninguna complicación. En los casos de hernias unilaterales fueron abordados mediante un r-TAPP, con tiempo quirúrgico promedio de 63 min (rango 35-70 minutos). Durante el procedimiento se

Tabla 1: Distribución por tipo de hernias.

\begin{tabular}{lc}
\multicolumn{2}{c}{ Tabla 1: Distribución por tipo de hernias. } \\
\hline Tipo de hernia & $\mathrm{n}(\%)$ \\
\hline Directas & $25(23 \%)$ \\
Indirectas & $53(42 \%)$ \\
Femorales & $15(11 \%)$ \\
Mixtas & $30(24 \%)$ \\
\hline
\end{tabular}


hizo una disección cuidadosa y retiro de la malla previa. No se reportaron complicaciones transoperatorias y todos los pacientes fueron egresados a las 24 horas.

El promedio de seguimiento fue de ocho meses, siendo el menor de seis meses y el mayor de 15 meses, ningún paciente se perdió en el seguimiento. Siete pacientes presentaron seroma en herida quirúrgica umbilical (7.14\%). No se presentaron otro tipo de complicaciones en ninguno de dichos pacientes. Son egresados entre 8-24 horas. Hasta el momento ningún paciente ha presentado recidiva.

\section{DISCUSIÓN}

La realización de una plastia inguinal abierta o laparoscópica representa un desafío para el cirujano general, a pesar de que es uno de los procedimientos quirúrgicos electivos más comunes en cirugía general, en Estados Unidos representa el $10-15 \%$ de todas las cirugías. ${ }^{10,11} \mathrm{La}$ técnica quirúrgica ha presentado cambios significativos en los últimos 27 años. Actualmente, existen dos tipos de reparación de hernias inguinales unilaterales, la reparación transabdominal preperitoneal (TAPP) y la reparación totalmente extraperitoneal (TEP), y en el caso de las hernias bilaterales se puede usar la técnica de Stoppa modificada. Las ventajas de la reparación robótica sobre la abierta son: reducción del dolor posoperatorio e inicio más temprano del trabajo y las actividades normales. ${ }^{12}$

A pesar de estas claras ventajas, el abordaje robótico ha tardado en ganar popularidad entre muchos cirujanos. La tecnología robótica ofrece a los cirujanos una visualización tridimensional, pinzas articuladas de $540^{\circ}$ de rotación, visión con zoom $10 \times$ (la visión laparoscópica ofrece un zoom $2 \times$ ), lo cual disminuye el riesgo de lesión nerviosa y vascular, aumento de la destreza, precisión y neutralización del temblor fino, permitiendo al cirujano realizar cirugías de mínimo acceso más complejas con mayor facilidad. ${ }^{13,14}$

Los resultados de este estudio indican que la reparación robótica de hernias es factible para todos los tipos de hernias (bilaterales, recurrentes, directas, indirectas e inguinoescrotales), ya que es una técnica segura y efectiva. El tiempo quirúrgico promedio en las plastias unilaterales fue de 46.3 minutos totales, y en las bilaterales y recurrentes de 72.3 minutos totales. Todos esos pacientes se egresaron a las 24 horas de realizarse el procedimiento, reportando dolor mínimo en el sitio quirúrgico.

El cirujano puede ofrecer reparación de hernias inguinales asistidas por robot en pacientes con índice de masa corporal (IMC) mucho más alto. ${ }^{15}$ Hay que considerar que los cirujanos poseedores de habilidad laparoscópica previa en reparación de hernias inguinales, al tener acceso a la tecnología robótica, pueden realizar casos complejos como hernias inguinoescrotales, recurrentes y bilaterales. ${ }^{16,17}$
Arcerito y colaboradores reportan en ambas técnicas quirúrgicas (r-TAPP y r-Stoppa modificada), una tasa baja de complicaciones intraoperatorias y baja tasa de conversión (0.03 y $1.4 \%$ respectivamente), en el posoperatorio también se asocia con tasas bajas de retención urinaria y seroma/hematoma ( $4.1 \%$ y $3.5 \%$ respectivamente), finalmente, la tasa de recurrencia es de $0.18 \% .{ }^{11}$ En la serie de casos reportada, sólo siete pacientes presentaron seroma en el puerto umbilical (7.14\%), no se presentaron otro tipo de complicaciones transoperatorias y en el seguimiento posoperatorio a siete días, 30 días, seis meses y un año se tiene una tasa de recurrencia del $0 \%$.

Una de las discusiones más antiguas, respecto a la cirugía robótica y en especial en la plastia inguinal asistida por robot, es su costo-efectividad. Sin embargo, dado el avance y la experiencia en este abordaje quirúrgico se han realizado modificaciones a la técnica que disminuyen los costos y en la experiencia de este grupo quirúrgico llegan casi a equipararse con aquellos del abordaje quirúrgico. Los paquetes quirúrgicos de las plastias inguinales en los hospitales, en donde este grupo labora, van entre los $\$ 108,000-120,000$ MXN sin importar si se requiere abordaje bilateral o unilateral, ya que no se requieren más insumos al usar una única malla en las reparaciones r-Stoppa y siendo ésta una de las grandes ventajas. Comparado contra los paquetes de cirugía laparoscópica que oscilan entre los \$103,000$\$ 115,000$ MXN, dependiendo de cuáles insumos son utilizados (número de tackers, tipos, etc.) y si el abordaje requiere reparación unilateral o bilateral, representando un costo aproximado de entre el 5-10\% menor cuando se compara con la cirugía robótica.

Estos hallazgos son corroborados por algunos otros autores, como Kimberly E. Waite quien en 2016 comparó 64 casos consecutivos (24 laparoscópicos y 39 robóticos) contrastando tiempos operativos, costos, dolor y tiempos de recuperación, encontrando que la cirugía robótica disminuye de manera significativa el dolor posoperatorio y los tiempos de recuperación; sin embargo, aumentan los tiempos quirúrgicos. En cuanto a los costos, únicamente se aumentó en el costo directo de $\$ 3,216$ a $\$ 3,479$ dólares. ${ }^{13}$

A pesar de tales hallazgos aquí reportados, existe abundante literatura en donde se cuestiona el abordaje quirúrgico, por la disponibilidad (en México únicamente existen 17 sistemas Da Vinci), y por aumento de costos, la mayoría de las series reportan un aumento de los costos en las plastias inguinales de entre un $30-40 \%{ }^{18-21}$

\section{CONCLUSIONES}

En esta serie se muestra seguridad, efectividad y reproducibilidad a corto plazo de las técnicas r-TAPP y r-Stoppa modificada para pacientes con hernias inguinales unilate- 
rales y bilaterales, respectivamente, con una morbilidad baja y mortalidad nula en nuestros pacientes.

Existen todavía algunas desventajas en el abordaje asistido por robot, como lo son la disponibilidad y el costo, pero se considera que la modificación de la técnica puede disminuir los costos; sin embargo, hacen falta estudios que evalúen dichos parámetros en México y las observaciones reportadas en cuanto a costos sólo aplican a hospitales privados.

Hacen falta mayores estudios, con seguimiento a mayor tiempo, que evalúen de manera comparativa las ventajas y/o desventajas de estos abordajes para las plastias inguinales en nuestro país.

\section{REFERENCIAS}

1. Podolsky D, Novitsky Y. Robotic inguinal hernia repair. Surg Clin N Am. 2020; 100: 409-415.

2. Tam V, Rogers DE, Al-Abbas A, Borrebach J, Dunn SA, Zureikat $\mathrm{AH}$ et al. Robotic inguinal hernia repair: a large health system's experience with the first 300 cases and review of the literature. J Surg Res. 2019; 235: 98-104.

3. Iraniha A, Peloquin J. Long-term quality of life and outcomes following robotic assisted TAPP inguinal hernia repair. J Robot Surg. 2018; 12: 261-269.

4. Kakiashvili E, Bez M, Abu Shakra I, Ganam S, Bickel A, Merei $\mathrm{F}$ et al. Robotic inguinal hernia repair: Is it a new era in the management of inguinal hernia? Asian J Surg. 2021; 44: 93-98.

5. Gamagami R, Dickens E, Gonzalez A, D’Amico L, Richardson C, Rabaza J et al. Open versus robotic-assisted transabdominal preperitoneal (R-TAPP) inguinal hernia repair: a multicenter matched analysis of clinical outcomes. Hernia. 2018; 22: 827-836.

6. Aiolfi A, Cavalli M, Micheletto G, Bruni PG, Lombardo F, Perali $C$ et al. Robotic inguinal hernia repair: is technology taking over? Systematic review and meta-analysis. Hernia. 2019; 23: 509-519.

7. Kudsi OY, McCarty JC, Paluvoi N, Mabardy AS. Transition from laparoscopic totally extraperitoneal inguinal hernia repair to robotic transabdominal preperitoneal inguinal hernia repair: a retrospective review of a single surgeon's experience. World J Surg. 2017; 41: 2251-2257.

8. Barbash GI, Glied SA. New technology and health care costs--the case of robot-assisted surgery. $N$ Engl J Med. 2010; 363: 701-714.
9. Pradarelli JC, Thornton JP, Dimick JB. Who is responsible for the safe introduction of new surgical technology?: an important legal precedent from the da vinci surgical system trials. JAMA Surg. 2017; 152: 717-718.

10. Escobar DJE, Gonzalez A, Donkor C. Robotic inguinal hernia repair. J Surg Oncol. 2015; 112: 310-314.

11. Arcerito M, Changchien E, Bernal O, Konkoly-Thege A, Moon J. Robotic inguinal hernia repair: technique and early experience. Am Surg. 2016; 82: 1014-1017.

12. Escobar DJE, Ramos MG, Seetharamaiah R, Donkor C, Rabaza J, González A. Feasibility of robotic inguinal hernia repair, a single institution experience. Surg Endosc. 2016; 30: 4042-4048.

13. Waite KE, Herman MA, Doyle PJ. Comparison of robotic versus laparoscopic transabdominal preperitoneal (TAPP) inguinal hernia repair. J Robot Surg. 2016; 10: 239-244.

14. Claus C, Furtado M, Malcher F, Cavazzola LT, Felix E. Ten golden rules for a safe MIS inguinal hernia repair using a new anatomical concept as a guide. Surg Endosc. 2020; 34: 1458-1464. doi: 10.1007/s00464-020-07449-z.

15. Kolachalam R, Dickens E, D'Amico L, Richardson C, Rabaza J, Gamagami R et al. Early outcomes of roboticassisted inguinal hernia repair in obese patients: a multiinstitutional, retrospective study. Surg Endosc. 2018; 32: 229-235. doi: 10.1007/s0464-017-5665-z.

16. Wu JJ, Way JA, Eslick GD, Cox MR. Transabdominal preperitoneal versus open repair for primary unilateral inguinal hernia: a meta-analysis. World J Surg. 2018; 42: 13041311.

17. Charles EJ, Mehaffey JH, Tache-Leon CA, Hallowell PT, Sawyer RG, Yang Z. Inguinal hernia repair: is there a benefit to using the robot? Surg Endosc. 2018; 32: 2131-2136.

18. Ito F, Jarrard D, Gould JC. Transabdominal preperitoneal robotic inguinal hernia repair. J Laparoendosc Adv Surg Tech A. 2008 ; 18: 397-399.

19. Cetrulo LN, Harmon J, Ortiz J, Canter D, Joshi AR. Case report of a robotic-assisted laparoscopic repair of a giant incarcerated recurrent inguinal hernia containing bladder and ureters. Int J Med Robot. 2015; 11 (1): 15-17.

20. Abdelmoaty WF, Dunst CM, Neighorn C, Swanstrom LL, Hammill CW. Robotic-assisted versus laparoscopic unilateral inguinal hernia repair: a comprehensive cost analysis. Surg Endosc. 2019; 33: 3436-3443. doi: 10.1007/s ${ }^{0} 0464-018-$ 06606-9.

21. Henriksen NA, Jensen KK, Muysoms F. Robot-assisted abdominal wall surgery: a systematic review of the literature and meta-analysis. Hernia. 2019; 23: 17-27. doi: 10.1007/ s'0029-018-1872-3. 